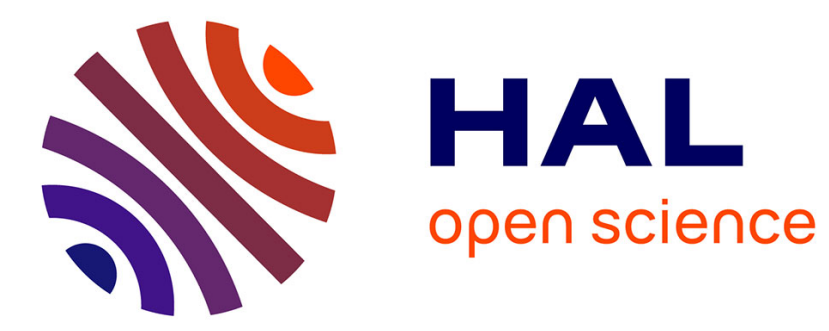

\title{
Quatre problèmes pour le choix libre
}

Jacques Jayez, Lucia Tovena

\section{To cite this version:}

Jacques Jayez, Lucia Tovena. Quatre problèmes pour le choix libre. Langue française, 2010, 166, pp.51-72. 10.3917/lf.166.0051 . hal-01069791

\section{HAL Id: hal-01069791 \\ https://hal.science/hal-01069791}

Submitted on 29 Sep 2014

HAL is a multi-disciplinary open access archive for the deposit and dissemination of scientific research documents, whether they are published or not. The documents may come from teaching and research institutions in France or abroad, or from public or private research centers.
L'archive ouverte pluridisciplinaire HAL, est destinée au dépôt et à la diffusion de documents scientifiques de niveau recherche, publiés ou non, émanant des établissements d'enseignement et de recherche français ou étrangers, des laboratoires publics ou privés. 


\title{
Quatre problèmes pour le choix libre
}

\author{
Jacques Jayez et Lucia M. Tovena
}

Résumé : Cet article fait le point sur quatre questions importantes qui sont encore en partie ouvertes. 1- Concernant les frontières mêmes de la notion de «choix libre ", il est proposé de caractériser le noyau du phénomène par une contrainte minimale d'équivalence dans une dimension. 2- L'élargissement, qui semble intuitivement correspondre à une notion très satisfaisante pour les TCL, est analysé comme un effet et rejeté en tant que phénomène explicatif. 3-Concernant la question de la valeur existentielle ou universelle des TCL, nous argumentons en faveur d'une position nuancée. 4- Enfin, les phrases comparatives constituent toujours une question ouverte, car elles peuvent abriter des TCL même lorsqu'elles font référence à des événements particuliers et sans les cantonner à une interprétation dépréciative. Mots clés :Sémantique, Choix libre, Monotonie, Comparatives, Elargissement, $n$ 'importe qu-

\begin{abstract}
This paper takes stock on four crucial issues concerning FCIs. 1- As for the definition of the phenomenon, it is proposed to characterise free choiceness via a minimal constraint enforcing equivalence along a dimension. 2- Widening, that offers an intuitively appealing way of describing the behaviour of FCI, is shown to be an effect rather than an explanatory phenomenon. 3- Tenets of the existential vs universal debate are recalled and argued to be non conclusive. 4- Comparatives are an unresolved puzzle, because their being episodic does not affect their suitability for hosting FCI in non-depreciative readings.
\end{abstract}

Keywords:Semantics, Free-Choice, Monotony, Comparatives, Widening, n’importe qu-

\section{Introduction}

Les termes dits "à choix libre », désormais TCL, sont essentiellement des déterminants comme n'importe quel ou des pronoms comme qui que ce soit. Ils indiquent qu'il y a une latitude de choix sur un ensemble de référents possibles. Dans cet article, nous aborderons quatre problèmes, débattus dans la littérature spécialisée, mais que nous considérons comme partiellement non résolus. Bien que ces problèmes ne soient pas toujours mutuellement indépendants, nous avons choisi, pour la commodité de la présentation, de les traiter dans des sections séparées. En voici la liste, avec à chaque fois une question générale qui les caractérise et un exemple qui les illustre.

A quoi correspond la « liberté de choix » suggérée par le nom des TCL? Par exemple, par rapport à une phrase comme Choisissez un sujet, supposée se rapporter à une situation d'examen, en quoi la variante Choisissez n'importe quel sujet est-elle différente? Nous traitons cette question dans la section 2.

Dans la section 3, nous examinons la question de l' «élargissement ». Ce terme, qui est la traduction littérale du widening de Kadmon et Landman (1993), renvoie à l'impression qu'une phrase utilisant un TCL couvre a priori un domaine plus vaste que la variante avec un indéfini sans choix libre. Par exemple, N'importe quel étudiant doit avoir une carte d'étudiant concernerait absolument tous les étudiants, sans aucune espèce de restriction, alors que la variante Un étudiant doit avoir une carte d'étudiant pourrait éventuellement exclure les étudiants qui, pour une raison ou une autre, sont considérés comme atypiques. Nous discutons une version récente de l'éargissement proposée par Chierchia (2006).

Dans la section 4 nous abordons la distinction existentiel-universel. Elle a été posée initialement pour l'anglais any mais concerne en fait la plupart des TCL qui ne sont pas clairement universels, comme tout. Par exemple, pour une phrase comme N'importe quel ouvrage peut être consulté, quel type d'analyse faut-il privilégier? Une analyse où n'importe quel ouvrage est l'équivalent de un ouvrage, mais avec des contraintes supplémentaires liées 
à sa nature de TCL et qui produiraient la lecture « tous les ouvrages peuvent être consultés »? $\mathrm{Ou}$ au contraire une analyse dans laquelle n'importe quel est, au moins dans ce type de contexte, intrinsèquement universel?

Enfin, la section 5 est consacrée au problème des TCL dans les phrases comparatives, telles que Paul a montré plus de technique que n'importe quel spécialiste de la boîte. Un des problèmes de ces phrases est qu'elles peuvent faire référence à un événement particulier, ce qui n'est pas toujours possible avec les TCL qu'elles contiennent, comme en témoigne le contraste avec ??Paul a battu n'importe quel spécialiste de la boîte.

\section{La notion de choix libre}

Bien que les limites exactes de cette notion soient encore l'objet de discussion, il est possible d'en donner une définition générale (mais vague). Un TCL concerne un ensemble d'entités et signale que «n'importe quelle » entité de cet ensemble peut être choisie comme possédant un certaine propriété. Comme on le verra, un TCL typique demande qu'il y ait un choix entre des options présentées comme équivalentes. De manière plus précise, si on analyse un énoncé dans les termes de la quantification généralisée on peut y distinguer une restriction $(\mathrm{R})$ et une portée (P), liées par un opérateur, dont le rôle est rempli par le TCL. Ainsi, dans l'exemple (1), la restriction $\mathrm{R}$ correspond au $\mathrm{N}$ complexe musicien ici présent et la portée $\mathrm{P}$ à la propriété d'être capable de lire une partition.

(1) $[\mathrm{N} \text { 'importe quel] [musicien ici présent }]_{\mathrm{R}}$ [est capable de déchiffrer une partition $]_{\mathrm{P}}$

(1) implique que tous les musiciens présents sont équivalents quant au choix de celui qui est capable de déchiffrer une partition. Cependant, n'importe quel n'est pas un quantificateur universel, comme le montre (2). Les paraphrases en tous les en (2b) et (2d) ne sont en effet pas équivalentes.

(2) a. Ouvre n'importe quelle porte

b. Ouvre toutes les portes

c. S'il y avait n'importe quel incident à la centrale, les écologistes se plaindraient

d. S'il y avait tous les incidents à la centrale, les écologistes se plaindraient

Il y a là une sorte de paradoxe, souvent relevé : bien qu'un TCL comme n'importe quel ne soit pas un quantificateur universel, son interprétation, au moins dans certains cas, est bien celle d'une quantification universelle. Comparons (1) et (3). A la différence de (1), (3) est bien moins naturelle que sa variante en tous les. Pourquoi ?

(3) ?? N'importe quel musicien ici présent a déchiffré une partition ce matin

La réponse est simplement qu'il n'y a plus de choix. Si tous les musiciens sont équivalents, il y a bien «liberté » au sens où nous pouvons tous les considérer comme des candidats potentiels pour satisfaire la portée (avoir déchiffré une partition ce matin), mais il n'y a pas de " choix » puisque tous, étant équivalents par rapport à la portée, ont déchiffré la partition. Le contraste avec (1) vient de ce que, dans ce dernier exemple, nous avons une structure modale cachée, liée à " être capable de ». Il a été souvent noté que les TCL sont à leur aise dans des interprétations modales de possibilité ou de permission. Une structure modale, habituellement considérée comme un ensemble d'alternatives, permet de réconcilier liberté et choix. Les individus ou les sous-ensembles de la restriction sont équivalents par rapport à la portée, mais l'existence de plusieurs alternatives autorise encore un certain type de choix, que nous allons 
préciser.

La manière dont un opérateur comme n'importe quel permet de construire l'interprétation universelle en (1) peut se décomposer en deux instructions, relatives à un ensemble imaginaire d'alternatives (Jayez \& Tovena 2005). Pour (1), ce sont toutes les situations où un des musiciens incriminés déchiffre une partition. Appelons $\mathrm{S}$ cet ensemble de situations.

(a) Dans l'ensemble des musiciens présents, aucun n'est exclu. Autrement dit, pour chaque musicien $\mathrm{m}$, il y a au moins une situation dans $\mathrm{S}$ telle que le musicien $\mathrm{m}$ déchiffre une partition dans S. Nous appellerons cette configuration une configuration PAS-DE-PERDANT.

(b) Dans l'ensemble des musiciens présents, aucun n'est privilégié. Autrement dit, il n'y a aucun musicien qui déchiffre une partition dans toutes les situations. Nous appellerons cette configuration une configuration PAS-DE-GAGNANT.

L'association PAS-DE-PERDANT + PAS-DE-GAGNANT crée une équivalence modale que nous appelons métaphoriquement équité. Parce qu'elle est modale ${ }^{1}$, l'équité permet un choix entre les alternatives. Il est essentiel de comprendre que ce choix ne concerne pas directement les individus. (1) implique que tous les musiciens présents ont la capacité de déchiffrer une partition. Il n'y a donc pas à choisir entre eux. En revanche, l'instanciation de cette capacité, c'est à dire les événements de déchiffrage peuvent concerner différents musiciens de l'ensemble de départ dans les différentes situations alternatives. Dès lors que nous sommes cantonnés à une situation particulière, comme en (3), la marge de manœuvre est insuffisante pour permettre un choix modal, certes fictionnel mais sémantiquement pertinent. De tels cas entraînent une violation de l'équité par suppression des alternatives d'un certain type, les alternatives référentielles, c'est-à-dire des situations qui représentent des états du monde réel dans le temps, par exemple ses possibles évolutions dans le futur. (3) illustre les interprétations épisodiques, où l'on fait référence à un événement simple ou complexe particulier.

En restant pour le moment dans le domaine des alternatives référentielles, il existe également des cas où on empêche ouvertement la variation modale entre les alternatives, même si celles-ci « existent », c'est-à-dire peuvent être associées à un opérateur modal. C'est le cas dans (4), puisque (4a) est incompatible avec PAS-DE-GAGNANT et (4c) avec PAS-DEPERDANT. On remarque que, dans les deux cas, un indéfini «simple » comme un ne crée aucun problème.

(4) a. ?? Vous êtes autorisé à annoter n'importe quelle partition, celle de Shostakovich

b. Vous êtes autorisé à annoter une partition, celle de Shostakovich

c. ?? Vous êtes autorisé à annoter n'importe quelle partition, qui ne doit pas être celle de Shostakovich

d. Vous êtes autorisé à annoter une partition, qui ne doit pas être celle de Shostakovich

La violation modale peut aussi arriver par le biais de la quantification universelle. En suivant (Tovena \& Jayez 1999), nous considérons tout déterminant comme un TCL et comme un quantificateur universel. Dans cette perspective, (5a) est très bizarre parce que les mêmes fichiers (tous ceux qui sont pertinents au moment de l'énonciation) sont consultés dans toutes les alternatives qui correspondent aux situations où l'allocutaire exploite la permission qui lui est offerte. Il s'agit d'une violation flagrante de PAS-DE-GAGNANT.

(5) a. *Consulte tout fichier; nous n'avons pas de secret pour toi

b. Tout fichier peut être consulté ; nous n'avons pas de secret pour toi

c. Consulte n'importe quel fichier; nous n'avons pas de secret pour toi

\footnotetext{
${ }^{1}$ Pour ne pas compliquer, nous admettrons pour le moment que l'équité est de nature modale, c'est-à-dire liée à la présence d'un opérateur modale. Nous généraliserons la notion dans la section 3, définition (12).
} 
L'intervention de plusieurs facteurs vient compliquer ce schéma de départ. Nous ne mentionnerons ici que celui de la dimension sémantique ou intervient l'équité, c'est-à-dire le type d'alternatives et d'opérateur modal considérés. Dans les exemples précédents, nous n'avons fait appel qu'à la dimension référentielle. Le TCL un quelconque illustre la dimension épistémique ${ }^{2}$, où les alternatives sont des états de croyance d'un agent. En (6a), le musicien a annoté une partition particulière, mais la phrase implique que le locuteur ignore laquelle. (6b) et (6c) montrent que le comportement de un quelconque est équivalent à celui de n'importe quel dans la dimension épistémique: l'interprétation choisie ne doit pas impliquer qu'un individu est exclu ou imposé dans l'ensemble des croyances du locuteur, ou, plus généralement de l'agent dont on adopte la perspective épistémique. En (6d), bien que le locuteur identifie le problème qui affecte la machine de Marie, l'adoption de la perspective de cette dernière suffit à rendre un quelconque acceptable.

(6) a. Le musicien a annoté une partition quelconque

b. ?? Le musicien a annoté une partition quelconque, celle de Shostakovich

c. ?? Le musicien a annoté une partition quelconque, pas celle de Shostakovich

d. Paul a effacé les fichiers système de Marie, et celle-ci s'est bien rendu compte qu'il y avait un problème quelconque sur sa machine

On remarquera qu'en général PAS-DE-PERDANT est une contrainte moins saillante que PASDE-GAGNANT et les interprétations exceptives demeurent possibles pour beaucoup de locuteurs $(7 \mathrm{a}-\mathrm{b})$. Toutefois, une interprétation exceptive peut être assimilée à une précision sur le domaine de la restriction et il est difficile de tirer des conclusions fermes de tels exemples. Il demeure possible que les deux contraintes n'aient pas la même force et une étude plus détaillée devrait apprécier leur importance pour chaque TCL.

(7) a. Tu peux choisir n'importe quelle partition sauf celle de Shostakovich

b. Tu peux choisir une partition quelconque qui ne soit pas celle de Shostakovich

Outre les dimensions référentielle et épistémique, on peut invoquer une dimension affective pour regrouper un certains nombres d'observations sur les TCL, sans préjuger de la possibilité de faire des distinctions plus fines. Cela concerne en particulier n'importe qu- en français, pour lequel on constate souvent des valeurs dépréciatives ou d'indifférence.

(8) a. Comme Paul était en retard pour son boulot, il a vite avalé n'importe quelle mixture avant de se remettre au travail

b. Comme Paul était paniqué il a fait un peu n'importe quoi

c. N'importe quel stylo fera l'affaire, j'ai juste besoin de signer

Comme l'a noté Vlachou (2007), des cas comme (8a,b) montrent que n'importe qu- est possible sous des interprétations épisodiques (Larrivée 2007, Muller 2007a, Paillard 1997). Toutefois, il faut préciser trois points. D'abord, il ne s'agit pas d'un mécanisme de compensation homogène. On pourrait imaginer que si, en tant que TCL, n'importe qu- a besoin de l'équité il peut la trouver dans n'importe quelle dimension, référentielle, épistémique ou affective, et que son allergie apparente pour les phrases épisodiques se manifeste uniquement avec les interprétations référentielles. Ce n'est pas le cas, car la dimension épistémique n'est pas suffisante pour rendre n'importe qu-compatible avec une

\footnotetext{
${ }^{2}$ Nous traitons uniquement ici de la structure un $\mathrm{N}$ quelconque. Il existe une autre structure un quelconque $\mathrm{N}$, qui ne semble pas entièrement superposable, mais qui relève aussi du choix libre.
} 
interprétation épisodique. Ainsi dans (9a), le locuteur peut parfaitement ignorer quel accident a frappé Marie. Cela ne suffit pas à rendre l'interprétation épisodique possible, alors qu'une variation modale, donc une interprétation non épisodique, ne pose aucun problème $(9 b)$.

(9) a. ??Marie a eu n'importe quel accident

b. Marie a pu avoir n'importe quel accident

On peut imaginer, compte tenu de l'origine diachronique de n'importe qu- (voir Béguelin 2002 et Pescarini 2009), que ce TCL communique l'idée qu'un agent n'opère aucun choix " sérieux », c'est-à-dire fondé sur un principe de choix quelconque. Ce serait alors cette valeur qui serait requise pour les interprétations épisodiques (Jayez et Tovena 2005). Lorsque la notion de choix intentionnel n'est pas pertinente, l'interprétation épisodique est très difficile (9a). Le caractère dépréciatif est associé à l'absence de choix intentionnel et paraît concerner au premier chef l'attitude de l'agent et, seulement de manière dérivée, le résultat de son action, comme le suggère (10), qui implique que Paul n'a pas répondu de manière bien contrôlée, mais que sa réponse était quand même satisfaisante.

(10) Paul a répondu n'importe quoi, et, par hasard, c'était ce qu'il fallait répondre !

Il faut ajouter que, dans ces cas, l'absence de choix fait partie du contenu principal et n'est pas une présupposition ou une implicatures (conventionnelle). On peut l'assimiler à un modifieur, sémantiquement sur le même plan qu'un adjectif ou qu'une relative, (10) signifiant «Paul a répondu [quelque chose] $]_{\text {ronom [qui était n'importe quoi] }}$ modifieur ». D'ailleurs, on trouve n'importe qui/quoi en position prédicative (C'est n'importe quoi), avec des adverbes qui modifient des SN prédicatifs (un peu, plutôt, largement, tout à fait) et la combinaison avec la négation produit exactement ce qu'on attend d'un groupe $\mathrm{N}+$ modifieur, Paul n'a pas répondu n'importe quoi pouvant s'analyser comme «Paul n'a pas répondu quelque chose qui était n'importe quoi », soit « Paul a répondu quelque chose et ce n'était pas n'importe quoi ». En résumé n'importe qui/quoi se comporte comme un pronom incorporant la valeur d'absence de choix. On trouve un comportement identique avec la négation pour n'importe quel $\mathrm{N}$. En revanche, on ne trouve pas un comportement prédicatif complet, cf. par exemple ?C'était un peu n'importe quel livre en face de C'était un peu n'importe quoi comme livre. Cela suggère que n'importe quel n'a pas (pas encore ?) le statut plein d'un déterminant avec modifieur du nom, autrement dit que n'importe quel $\mathrm{N}$ n'est pas équivalent à « Dét $\mathrm{N}$ qui est n'importe qui/quoi ».

Le deuxième point à préciser touche à l'indépendance des dimensions. En un sens, il y a presque toujours une interprétation intellectuelle (ignorance, équivalence théorique) ou affective (indifférence, dépréciation) des TCL. Si un TCL signale qu'il y a ou qu'il y aurait eu possibilité de choisir un élément quelconque de la restriction, cette possibilité est immédiatement rapportée à une raison explicite ou implicite, qui est propre au locuteur, à l'agent de l'action ou à une instance plus abstraite (des possibilités légales, par exemple). Devons nous en conclure que, au fond, «tout est lié »? Dans la dimension référentielle, nous avons évoqué (a) des interprétations modales, généralement associées à des opérateurs modaux explicites (impératif, futur et verbes modaux en particulier) et (b) des interprétations épisodiques, qui bloquent les TCL parce qu'elles empêchent l'équité. Dans la dimension épistémique, nous avons rencontré des alternatives épistémiques, qui ne sont pas concernées par la référence. Cela signifie qu'il est parfaitement possible d'avoir une seule alternative référentielle (le monde tel qu'il est) ou une impossibilité de variation à travers plusieurs alternatives et, en même temps, plusieurs alternatives épistémiques. On a donc deux dimensions orthogonales. Cette remarque s'applique également à la dimension affective, dont 
les alternatives, qui représentent les diverses préférences par rapport à certaines critères (la désirabilité pour un agent par exemple), n'interfèrent pas avec d'autres catégories d'alternatives. En effet, le fait qu'il existe une ou plusieurs alternatives référentielles ou épistémiques n'affecte pas l'existence ou l'absence d'alternatives affectives. Tout ce qu'on peut dire est que, en général, signaler l'existence d'alternatives référentielles se laisse rapporter en plus à des croyances ou à des préférences, et donc à des alternatives épistémiques ou affectives.

Le dernier point que nous aborderons concerne le caractère hypothétique ou contrefactuel (Choi 2007) de l'équivalence. Pour les alternatives référentielles ou épistémiques, on a plusieurs événements possibles ou envisageables. Pour les alternatives affectives, ce n'est pas nécessairement le cas, mais, de toute façon, l'intuition est que tout événement envisagé aurait pu être remplacé par un événement équivalent, l'absence de choix fondé sur des préférences conduisant à une réalisation aléatoire. Mais que représentent les alternatives dans cette interprétation « aléatoire »? Considérons à nouveau l'exemple (10). Intuitivement, il nous dit que l'identité de la réponse n'est pas significative et donc qu'on aurait pu tout aussi bien imaginer une autre réponse. En termes de probabilité, cela correspond à une loi uniforme. Comment l'exprimer? Si nous formulons une contrainte en termes de préférence, ce qui est techniquement possible, nous n'aurons qu'une relation indirecte et relativement complexe à nos deux contraintes de départ, PAS-DE-GAGNANT et PAS-DE-PERDANT. Une approche plus simple consiste à considérer des alternatives qui sont les résultats des préférences. Chaque alternative correspond à une situation préférée. L'équivalence entre les réponses dans le cas (10) correspond au fait qu'aucune réponse n'est privilégiée (vraie dans toutes les alternatives préférées) ou exclue (fausse dans toutes les alternatives préférées). Lorsqu'on sait qu'un événement spécifique s'est réalisé, on a une lecture contrefactuelle : une certaine réponse a effectivement été donnée, mais tout membre de l'ensemble de réponse se trouve à égalité avec cette réponse dans l'ensemble des réponses, et aurait pu être sélectionné à sa place, sans différence notable par rapport aux préférences.

Cette approche nous permet d'intégrer un cas un peu plus exotique, celui du subtrigging (LeGrand 1975), où un TCL normalement exclu se trouve amélioré par le biais d'une modification du nom sur lequel il porte. Ainsi (11b) est bien meilleur que (11a).

\section{(11) a. ??Tout étudiant a été renvoyé \\ b. Tout étudiant qui avait triché a été renvoyé \\ c. ??Tout étudiant qui était dans la pièce a entendu l'explosion}

Nous avons défendu ailleurs l'idée que, dans ce type de contraste, la dépendance des notions joue un rôle essentiel. En gros, (11b) est meilleure parce qu'elle évoque une dépendance entre deux propriétés, celle d'être un étudiant tricheur et celle d'être renvoyé. Elle se laisse alors interpréter comme contrefactuelle par rapport à l'identité des étudiants tricheurs (Jayez \& Tovena 2005, 2007). En d'autres termes, (11b) asserte que tous les étudiants qui avaient triché ont été renvoyés, et, en tant que telle, est épisodique, mais sous-entend qu'il s'agit de l'application d'une règle, peut-être liée à la situation, mais qui, en tant que règle, se serait appliquée à d'autres étudiants s'ils avaient triché dans les mêmes circonstances. D'une manière générale, ces exemples sont reçus de manière variable parce qu'il est plus ou moins facile d'imaginer une dépendance de propriété. Ainsi (11c) est en général senti comme bizarre car on a du mal à concevoir une dépendance claire entre les notions (qu'est-ce qu'entendre une explosion a à voir avec la propriété d'être un étudiant dans une certaine pièce ?). Nous poserons que les alternatives nécessaires pour le subtrigging sont des situations qui ressemblent suffisamment à la situation décrite pour que des événements comparables s'y produisent, mais où l'identité des individus qui vérifient la restriction (les étudiants tricheurs 
de (11b)) varie. Le statut de n'importe quel par rapport au subtrigging est compliqué par le fait que, dans une interprétation épisodique, n'importe quel déclenche une interprétation d'absence de choix peu compatible avec une dépendance de propriétés. En général, les exemples de subtrigging avec n'importe quel sont sentis comme peu naturels.

A partir de ces quelques remarques, on peut remodeler la définition de l'équité de la manière suivante. On conserve l'idée des contraintes PAS-DE-PERDANT et PAS-DE-GAGNANT qui assurent une égalité entre les membres de la restriction et on tient compte de la diversité des cas en faisant varier la nature des alternatives. La définition (12) explicite cette nouvelle version et le tableau (13) fait la liste des catégories d'alternatives.

(12) Soit R la restriction, P la portée. Pour une forme TCL R P, une interprétation préserve l'équité lorsqu'elle respecte PAS-DE-PERDANT et PAS-DE-GAGNANT sur l'ensemble des alternatives que le TCL utilise pour ce type d'interprétation.

(13) Ontologie des alternatives pour quelques TCL (les colonnes contiennent les termes possibles et leur caractérisation)

\begin{tabular}{|l|l|l|l|l|}
\hline TCL possibles & N'importe qu-, tout & Un quelconque & N'importe qu- & Tout \\
\hline Contraintes & \multicolumn{2}{|c|}{ PAS-DE-GAGNANT et/ou PAS-DE-PERDANT } \\
\hline Alternatives & du monde réel & épistémiques & variantes aléatoires & $\begin{array}{l}\text { variantes sur } \\
\text { la restriction }\end{array}$ \\
\hline Interprétation & Modale & épistémique & pas de choix sérieux & $\begin{array}{l}\text { dépendance } \\
\text { de propriétés }\end{array}$ \\
\hline $\begin{array}{l}\text { Int. épisodique } \\
\text { possible }\end{array}$ & Non & oui & oui & oui \\
\hline
\end{tabular}

Bien entendu, ce tableau ne concerne qu'un nombre limité d'éléments. Manquent par exemple quiconque, tous ceux qui utilisent des affixes comme que ce soit (qu-que ce soit, quelque $\mathrm{N}$ que ce soit) ou quel qu'il soit (dans la structure Dét[+indéfini] N quel qu'il soit). Nous renvoyons à (Muller 2006, 2007a) pour un panorama complet. La plupart de ces éléments servent à indiquer que tous les membres de la restriction sont concernés. Lorsqu'ils ont une valeur emphatique (par exemple il n'a vu personne comparé à il n'a vu personne que ce soit), on pourrait estimer qu'ils relèvent des TCL. C'est moins évident pour quiconque qui relève des tournures corrélatives et dont on devrait préalablement démontrer qu'il fait quelque chose de plus qu'un quantificateur universel comme tous les. Le cas de quelque est en partie analogue à celui de un quelconque mais demande une analyse que nous ne pouvons présenter ici. (Corblin 2005, Muller 2007b, Jayez \& Tovena 2008, et Falaus 2009 pour le roumain)

\section{L’élargissement}

Le terme élargissement correspond au terme widening, utilisé par Kadmon et Landman (1993) à propos de l'anglais any. Leur intuition initiale est que any est un indéfini existentiel et fait référence à un domaine de restriction maximal, pouvant inclure des individus atypiques ou marginaux. Ils proposent une contrainte selon laquelle l'utilisation de any doit conduire à un énoncé qui impliquerait l'énoncé alternatif avec un indéfini sans élargissement. Nous notons D+ le domaine élargi et D le domaine "standard». Par exemple, (14a) est exclue parce que sa forme logique (avec $\mathrm{D}+$ ) n'implique pas la forme logique associée à la version avec un indéfini sans élargissement (avec D), voir (14b). Inversement, (14c) est possible parce qu'il y a bien une implication de ce type, voir (14d). Les énoncé génériques seraient également possibles, si l'on accepte l'idée que any, bien qu'existentiel, est compatible avec une force de quantification universelle dans de telles interprétations, voir (14e-f). 
(14) a. *Paul read any book

b. $\exists x \in D_{\text {book }}+($ Paul-read(x) $)=/=>\exists x \in D_{\text {book }}($ Paul-read(x) $)$

c. Paul did not read any book

d. $\neg \exists \mathrm{x} \in \mathrm{D}_{\text {book }}+(\operatorname{Paul}-\operatorname{read}(\mathrm{x})) \Rightarrow \neg \exists \mathrm{x} \in \mathrm{D}_{\text {book }}(\operatorname{Paul}-\operatorname{read}(\mathrm{x}))$

e. Any book can be interesting

f. $\forall \mathrm{x} \in \mathrm{D}_{\text {book }}+$ (can-be-interesting $\left.(\mathrm{x})\right) \Rightarrow \forall \mathrm{x} \in \mathrm{D}_{\text {book }}$ (can-be-interesting $(\mathrm{x})$ )

Nous avons discuté cette notion d'élargissement dans (Jayez et Tovena 2005b) et nous nous bornerons à mentionner deux points. Premièrement, il est difficile de voir en quoi il y a élargissement du domaine lorsqu'on spécifie un ensemble de départ bien circonscrit, comme dans You may pick any card of this pack. Deuxièmement, l'hypothèse de Kadmon et Landman repose étroitement sur l'idée que any est existentiel. Ce que l'on doit faire dans le cas de tout reste du coup extrêmement obscur ; faut-il lui refuser l'élargissement, et donc admettre que les TCL sont profondément hétérogènes, ou lui refuser le statut de TCL, ce qui nécessite des arguments indépendants et moins sensibles à une théorie particulière?

Nous nous concentrerons sur le traitement plus récent proposé par Chierchia (2006), qui cherche à rendre compte du fait que, dans de nombreuses langues, les mêmes formes servent de TCL et de termes de polarité négative. Comme beaucoup de travaux récents en sémantique, celui de Chierchia exploite l'intuition selon laquelle, lorsqu'on communique un aspect particulier, on communique du même coup qu'on exclut ou, tout au moins, qu'on minimise les aspects concurrents. Cette intuition prend ses racines chez Grice avec, par exemple, la maxime de quantité, qui précise qu'un énoncé doit être interprété comme le plus fort possible étant donné le contexte et les connaissances du locuteur. Cela a pour conséquence qu'un énoncé communique implicitement que les énoncés alternatifs plus forts sont incertains ou faux. Par exemple, Paul a deux enfants implicite que Paul n'en a pas plus de deux. Dans l'approche de Chierchia, la hiérarchie de force correspond simplement à la hiérarchie implicative ( $\mathrm{si} \mathrm{A} \Rightarrow \mathrm{B}, \mathrm{A}$ est plus fort que $\mathrm{B}^{3}$ ). La stratégie suivie consiste à associer à des classes d'éléments lexicaux des implicatures spécifiques, qui font partie intégrante du fonctionnement grammatical, et, à ce titre, ne sont pas annulables par le contexte. D'une manière générale, l'implicature d'une expression A consiste à sélectionner un sous-ensemble maximal de l'ensemble des alternatives de A et à l'ajouter à A pour obtenir un enrichissement de A. Par exemple, Paul a deux enfants aura comme enrichissement \{Paul a deux enfants, Paul a un enfant $\}$, c'est-à-dire la proposition de départ plus toutes celles qu'elle implique, à savoir l'unique proposition Paul a un enfant. Les autres propositions alternatives (Paul a trois enfants, Paul a quatre enfants,...) seront considérées comme fausses.

Dans ce qui suit, pour des raisons de place, nous considérerons seulement deux des cas analysés par Chierchia, celui des termes à polarité négative (TPN) et celui des TCL universels (TCL-U). Avant de donner le détail de la proposition de Chierchia pour les TPN, nous la résumerons intuitivement. Imaginons une structure de forme Paul a résolu Dét problème où Dét est un déterminant indéfini et en même temps un TPN. Cette structure asserte que Paul a résolu un problème de l'ensemble des problèmes et a comme alternatives toutes les propositions qui portent sur un sous-ensemble maximal de l'ensemble des problèmes, indiqué par X, de forme Paul a résolu un problème de X. Toutes ces alternatives sont considérées comme fausses, car plus fortes que (impliquant) la proposition de départ. Mais ce résultat est contradictoire car, si Paul n'a pas résolu un problème d'au moins un sous-ensemble maximal de l'ensemble des problèmes, il n'a en fait résolu aucun problème. C'est pourquoi les TPN demandent un environnement qui élimine la concurrence entre proposition centrale et

\footnotetext{
${ }^{3}$ Cela recouvre en fait deux cas: celui de la logique propositionnelle où $\mathrm{A}$ «implique » $\mathrm{B}$ au sens de l'implication matérielle traditionnelle, et celui où $\mathrm{A} \subseteq \mathrm{B}$, lorsque $\mathrm{A}$ et $\mathrm{B}$ sont ou correspondent à des ensembles.
} 
alternatives : si Paul n'a pas résolu un problème, au sens de aucun problème, cela implique qu'il n'a résolu aucun problème d'aucun sous-ensemble (maximal) de l'ensemble des problèmes, et toutes les propositions correspondantes sont, non pas des alternatives, mais de simples conséquences de la proposition centrale.

Chierchia attribue aux TCL comme any une capacité d'élargissement, et cela en deux sens. Il distingue d'abord un domaine d'individus visé, en général saillant dans le contexte, dont la restriction forme un sous-ensemble. Par exemple, any boy renvoie à un domaine d'individus $\mathrm{D}$ à l'intérieur duquel on prélève les garçons. L'élargissement nous fait considérer des domaines $\mathrm{D}$ maximaux, ce qui veut dire qu'une proposition de forme any boy $\mathrm{P}$, où $\mathrm{P}$ est une propriété quelconque, attribue $\mathrm{P}$ à un garçon dans le domaine maximal visé, pas seulement dans un de ses sous-ensembles. Deuxièmement, un domaine visé n'est pas forcément local à un monde. De la définition formelle (pp. 578-579), il ressort qu'un domaine D est simplement un sous-ensemble d'individus de l'univers total des individus $U$, et pour chaque monde $\mathrm{w}, \mathrm{D}_{\mathrm{w}}$ est l'ensemble des éléments de D qui existent dans w. Nous admettrons qu'un domaine est l'extension globale d'une propriété $\mathrm{D}$, et nous noterons $\mathrm{D}_{U}$ l'union des ensembles qui vérifient $\mathrm{D}$ dans un monde ${ }^{4}$.

Comme Chierchia veut produire une théorie compositionnelle de l'élargissement, il a besoin de pouvoir composer les enrichissements. La composition des enrichissements est définie en (15). $\mathrm{x}$ et y sont des expressions de surface (mots ou séquences de mots), $[[\mathrm{x}]]$ note l'interprétation d'une expression $\mathrm{x}$. $\mathrm{a}(\mathrm{b})$ note l'application de la fonction a à l'argument $\mathrm{b}$. $\operatorname{enr}([[\mathrm{x}]])$ note l'enrichissement ${ }^{5}$ de $[[\mathrm{x}]]$, qui est « vide » dans la plupart des cas $($ enr $([[\mathrm{x}]])=$ $[[x]])$. L'existence de deux cas en (15) correspond à l'intuition suivante. Par définition, un opérateur Op monotone décroissant ${ }^{6}$ vérifie : $A$ et $B$ étant des propositions, si $\mathrm{Op}(\mathrm{B})$ et $\mathrm{A} \Rightarrow$ $\mathrm{B}$, alors $\mathrm{Op}(\mathrm{A})$. Si on se limite au cas des propositions, on voit que enr([[Op]](B)) renvoie $[[\mathrm{Op}]](\mathrm{B})$ plus toutes les propositions $[[\mathrm{Op}]](\mathrm{A})$ avec $\mathrm{A} \Rightarrow \mathrm{B}$. Si, au contraire, on applique l'enrichissement séparément à Op et à son argument $\mathrm{B}$, en admettant que enr $([[\mathrm{Op}]])=[[\mathrm{Op}]]$ (enrichissement vide), on obtient [[Op]](enr(B)), c'est-à-dire [[Op]](B) plus toutes les propositions $[[\mathrm{Op}]](\mathrm{C})$ telle que $\mathrm{B} \Rightarrow \mathrm{C}$. Le premier ensemble implique le second. La formulation de (15) équivaut donc à sélectionner l'interprétation la plus forte. Il s'agit pour Chierchia d'un principe général : lorsqu'il y a plusieurs possibilités, la composition doit être agencée pour obtenir l'interprétation la plus forte.

\section{(15) Enrichissement}

L'enrichissement d'une expression de forme de surface $\mathrm{x} y$, noté enr([[x y]]) est défini par:

- enr([[x]])(enr([[y]])), si x n'est pas monotone décroissant, et

$-\operatorname{enr}([[\mathrm{x}]]([[\mathrm{y}]]))$, sinon.

Les diverses classes de déterminants que Chierchia analyse se distinguent par leurs alternatives et par la fonction enr(). Pour les TPN, l'implicature correspond à ce qu'on nomme une exhaustification. L'assertion est existentielle (il existe au moins un individu qui vérifie la restriction dans un monde et la portée dans le monde d'évaluation). Les alternatives sont

\footnotetext{
${ }^{4} \mathrm{Si} \mathrm{W}$ est l'ensemble des mondes et $\mathrm{U}$ l'univers des individus, $\mathrm{D}_{\mathrm{U}}=\cup\left\{\mathrm{X}: \exists \mathrm{w} \in \mathrm{W}\left([[\mathrm{D}]]_{\mathrm{w}}=\mathrm{X}\right)\right\}$. La caractérisation donnée dans l'article est selon nous inutilement obscure, car elle mélange une notion extensionnelle (D comme ensemble) et une notion intensionnelle (D comme propriété à extension variable selon les différents mondes).

${ }^{5}$ Nous avons utilisé une présentation uniformément fonctionnelle, pour éviter le mélange entre indices et opérateur constaté dans le texte original.

${ }^{6}$ Ce qui correspond linguistiquement par exemple à une marque de négation ou d'interrogation, la prémisse d'une conditionnelle, ou un verbe « négatif » tel que douter, refuser, etc.
} 
toutes les variations de domaine à l'intérieur du domaine de la restriction, c'est à dire, en gros, toutes les expressions $\exists x\left(\mathrm{R}^{\prime}(\mathrm{x}) \& \mathrm{P}(\mathrm{x})\right)$ où $\mathrm{R}^{\prime} \subset \mathrm{R}$. L'enrichissement lui-même consiste en la proposition initiale plus toutes les alternatives qu'elle implique. La forme $[[\mathrm{A}]]_{\mathrm{m}}$, où $\mathrm{m}$ est un monde, est l'interprétation de $\mathrm{A}$ dans $\mathrm{m}$, soit une valeur de vérité si A est propositionnelle. La forme $\mathrm{Q}_{\mathrm{m}}(\mathrm{x})$, où $\mathrm{Q}$ est une propriété et $\mathrm{m}$ un monde est une abréviation pour $[[\mathrm{Q}(\mathrm{x})]]_{\mathrm{m}}=$ Vrai. $\operatorname{MAX}^{c}(\mathrm{X})$ dénote l'ensemble des sous-ensembles stricts maximaux de X.

\section{(16) Exhaustification pour les TPN}

1. [[TPN R P] $]_{D, w}=\exists w^{\prime} \exists x\left(x \in D_{w} \& R_{w},(x) \& P_{w}(x)\right)$

2. $\operatorname{ALT}\left([[T P N R P]]_{D, w}\right)=\left\{\exists w^{\prime} \exists x\left(x \in D^{\prime}{ }_{w}, \& R_{w}(x) \& P_{w}(x)\right): D^{\prime} \subset D^{\prime} \& D^{\prime} \in\right.$ $\operatorname{MAX}(\mathrm{D})\}$

3. $\operatorname{enr}\left([[T P N R P]]_{D, w}\right)=\left\{[[T P N R P]]_{D, w}\right\} \cup\left\{p: p \in A L T\left([[T P N R P]]_{D, w}\right) \&[[T P N R\right.$ $\left.\mathrm{P}]]_{\mathrm{D}, \mathrm{w}} \Rightarrow \mathrm{p}\right\}$

Comme on l'a signalé plus haut, toutes les alternatives qui ne font pas partie de l'enrichissement sont considérées comme fausses. Or, on n'a jamais :

$$
\exists \mathrm{w}^{\prime} \exists \mathrm{x}\left(\mathrm{x} \in \mathrm{D}_{\mathrm{w}}, \& \mathrm{R}_{\mathrm{w}}(\mathrm{x}) \& \mathrm{P}_{\mathrm{w}}(\mathrm{x})\right) \Rightarrow \alpha \text {, pour } \alpha \in \operatorname{ALT}\left([[\mathrm{TPN} R \mathrm{P}]]_{\mathrm{D}, \mathrm{w}}\right) \text {. }
$$

En effet, si un individu de $\mathrm{D}_{\mathrm{w}}$ ' satisfait $\mathrm{R}$ dans $\mathrm{w}$ ' et $\mathrm{P}$ dans $\mathrm{w}$, il ne s'ensuit pas qu'un individu de $\mathrm{D}^{\prime}{ }_{\mathrm{w}}$, $\subset \mathrm{D}_{\mathrm{w}}$ ' satisfasse $\mathrm{R}$ dans $\mathrm{w}^{\prime}$ et $\mathrm{P}$ dans $\mathrm{w}$. En cumulant toutes les alternatives rejetées, on obtient, pour chaque $\mathrm{w}^{\prime}$ tel que $\exists \mathrm{x}\left(\mathrm{x} \in \mathrm{D}_{\mathrm{w}}, \& \mathrm{R}_{\mathrm{w}}(\mathrm{x}) \& \mathrm{P}_{\mathrm{w}}(\mathrm{x})\right)$, la négation de toute alternative pour tout sous-ensemble maximal de $\mathrm{D}_{\mathrm{w}}$, soit :

$$
\neg \exists D^{\prime}{ }_{w}, \exists x\left(D^{\prime} \subset D \& D^{\prime} \in \operatorname{MAX}^{\prime}(D) \& x \in D^{\prime}{ }_{w}, \& R_{w},(x) \& P_{w}(x)\right)
$$

Cela produit une contradiction si l'opérateur d'enrichissement est appliqué directement à $[[T P N \text { R P }]]_{D, w}$. En revanche, lorsqu'on a un enchâssement dans un opérateur monotonedécroissant Op, l'enrichissement a une forme enr([[Op]]([[TPN R P] $\left.\left.]_{\mathrm{D}, \mathrm{w}}\right)\right)$, avec des alternatives de forme $[[\mathrm{Op}]]\left(\exists \mathrm{w}^{\prime} \exists \mathrm{x}\left(\mathrm{x} \in \mathrm{D}^{\prime}{ }_{\mathrm{w}}, \& \mathrm{R}_{\mathrm{w}},(\mathrm{x}) \& \mathrm{P}_{\mathrm{w}}(\mathrm{x})\right): \mathrm{D}^{\prime} \subset \mathrm{D} \& \mathrm{D}^{\prime} \in\right.$ $\operatorname{MAX}(\mathrm{D}))$ pour chaque $\mathrm{D}^{\prime}$. Dans le cas d'une négation NEG, par exemple, où on posera $[[N E G]]=\neg$, l'exhaustification ne donne lieu à aucune contradiction, puisqu'on a $\neg \exists$ w' $\exists x$ (x $\left.\in \mathrm{D}_{\mathrm{w}}, \& \mathrm{R}_{\mathrm{w}}{ }^{\prime}(\mathrm{x}) \& \mathrm{P}_{\mathrm{w}}(\mathrm{x})\right)$ plus $\neg \exists \mathrm{D}^{\prime}{ }_{\mathrm{w}}, \exists \mathrm{x}\left(\mathrm{D}^{\prime} \subset \mathrm{D} \& \mathrm{D}{ }^{\prime} \in \operatorname{MAX}(\mathrm{D}) \& \mathrm{x} \in \mathrm{D}^{\prime}{ }_{\mathrm{w}}{ }^{\prime} \& \mathrm{R}_{\mathrm{w}}{ }^{\prime}(\mathrm{x}) \&\right.$ $\left.\mathrm{P}_{\mathrm{w}}(\mathrm{x})\right)$, pour chaque $\mathrm{w}$.

Cette différence pourrait rendre compte d'asymétries comme *Paul a résolu quelque problème que ce soit et Paul n'a pas résolu quelque problème que ce soit. A ce stade, nous ferons deux remarques. D'abord, quelle trace intuitive de l'élargissement a-t-on pour any? Dans un environnement négatif, any n'est pas ressenti comme particulièrement emphatique et rien dans sa morphologie ne rappelle une variation libre, à la différence des mots en ever ou en que ce soit ou qu'il soit en français. Deuxièmement, l'analyse de Chierchia repose sur ce qu'on appelle dans la littérature sur les implicatures le "saut épistémique » (epistemic step) d'après Sauerland (2005). ${ }^{7}$ Ce saut consiste à passer d'une proposition de forme « $\mathrm{x}$ ne croit pas que $p$ » à une proposition de forme « $x$ croit que non-p». Dans le cas des TCL, on pourrait soutenir qu'une des manières de sauver les exemples épisodiques assertifs est de renoncer au saut épistémique, qui n'est jamais qu'une stratégie d'interprétation activée par défaut. Par exemple *Paul a résolu quelque problème que ce soit indiquerait que le locuteur asserte que Paul a résolu un problème, à choisir dans un ensemble maximal, et que la maximalité n'est que le reflet de son ignorance : le locuteur n'est tout simplement pas en mesure d'être plus précis. Cela n'est pas en soi étranger aux TCL puisque, comme on l'a vu, un quelconque exploite la dimension de l'ignorance. Pour que cette solution interprétative soit exclue, il faut indiquer que le saut épistémique est obligatoire, ou, ce qui revient au même

\footnotetext{
${ }^{7}$ Une idée analogue avait été proposée in (Tovena 2000) pour le phénomène de neg-raising.
} 
pour ce type d'exemple, que la dimension de l'ignorance n'est pas utilisable. Cela implique que any contient dans sa sémantique une information à cet effet. Du coup, le caractère très général revendiqué par Chierchia pour son analyse est moins évident, puisqu'il reste nécessaire d'indiquer la ou les dimensions que les TCL peuvent exploiter, information qui ne découle pas d'un système par implicatures.

Qu'en est-il pour les TCL à valeur universelle, c'est-à-dire pour nous n'importe quel ${ }^{8}$ ? L'intuition de Chierchia est que ces éléments sont gouvernés par une contrainte d'antiexhaustification, symétrique de la contrainte (16). Considérons une phrase de forme Paul a résolu TCL-U problème. Elle asserte que Paul a résolu un problème de l'ensemble des problèmes et détermine comme alternatives toutes les propositions semblables mais restreintes aux sous-ensembles de l'ensemble des problèmes. Chaque alternative peut être renforcée et implique alors la négation de l'alternative portant sur l'ensemble complémentaire. Si toutes ces négations étaient vraies, on aboutirait à une contradiction car Paul a résolu un problème sans avoir résolu aucun problème d'aucun sous-ensemble de l'ensemble des problèmes. On pose donc la négation des renforcements des alternatives, ce qui aboutit à une lecture universelle : Paul a résolu un problème de chaque sous-ensemble de l'ensemble des problèmes.

Dans le détail, contrairement à (16), l'anti-exhaustification (18) exclut le renforcement de la proposition initiale. Elle comporte deux aspects essentiels. D'une part les sous-ensembles de $\mathrm{D}$ retenus sont tous les sous-ensembles non vides, alors que c'étaient les sous-ensembles maximaux de D (D' $\in \operatorname{MAX}(\mathrm{D}))$ pour l'exhaustification. De ce fait les alternatives sont toutes les propositions qui assertent qu'un individu d'un D' $\subset$ D vérifie la restriction dans un monde et la portée dans le monde courant. D'autre part, l'enrichissement ne porte pas sur la proposition assertée mais sur ses alternatives, un point sur lequel nous reviendrons. L'enrichissement des alternatives est défini en (17) comme l'alternative plus la négation de chaque proposition qui asserte qu'un élément du complémentaire de D' dans D vérifie la restriction dans un monde et la portée dans le monde courant.

$$
\begin{aligned}
& \operatorname{enr}\left(\left\{\exists \mathrm{w}^{\prime} \exists \mathrm{x}\left(\mathrm{x} \in \mathrm{D}^{\prime}{ }_{\mathrm{w}}, \& \mathrm{R}_{\mathrm{w}},(\mathrm{x}) \& \mathrm{P}_{\mathrm{w}}(\mathrm{x})\right): \mathrm{D}^{\prime} \subset \mathrm{D}\right\}\right)=\left\{\exists \mathrm { w } ^ { \prime } \exists \mathrm { x } \left(\mathrm{x} \in \mathrm{D}^{\prime}{ }_{\mathrm{w}}, \& \mathrm{R}_{\mathrm{w}}{ }^{\prime}(\mathrm{x}) \&\right.\right. \\
& \left.\left.\mathrm{P}_{\mathrm{w}}(\mathrm{x})\right) \& \neg \exists \mathrm{w}^{\prime} \exists \mathrm{x}\left(\mathrm{x} \in \mathrm{D}^{\prime}{ }^{\prime}{ }_{\mathrm{w}}, \& \mathrm{R}_{\mathrm{w}},(\mathrm{x}) \& \mathrm{P}_{\mathrm{w}}(\mathrm{x})\right): \mathrm{D}^{\prime} \subset \mathrm{D} \& \mathrm{D}^{\prime}, \subseteq \mathrm{D}-\mathrm{D}^{\prime}\right\}
\end{aligned}
$$

Finalement, l'anti-exhaustification pour les TCL-U est définie en (18). Elle pose le même contenu que l'exhaustification, mais les alternatives concernent tous les sous-ensembles de D, pas seulement les sous-ensembles maximaux et, surtout, l'enrichissement consiste à nier les enrichissements des alternatives.

\section{(18) Anti-exhaustification pour les TCLU}

1. [ [TCLU R P] $]_{D, w}=\exists w^{\prime} \exists x\left(x \in D_{w}, \& R_{w},(x) \& P_{w}(x)\right)$

2. $\operatorname{ALT}\left([[T C L U R P]]_{D, w}\right)=\left\{\exists \mathrm{w}^{\prime} \exists \mathrm{x}\left(\mathrm{x} \in \mathrm{D}^{\prime}{ }_{\mathrm{w}}, \& \mathrm{R}_{\mathrm{w}},(\mathrm{x}) \& \mathrm{P}_{\mathrm{w}}(\mathrm{x})\right): \mathrm{D}^{\prime} \subset \mathrm{D}\right\}$

3. enr $\left([[T C L U R P]]_{D, w}\right)=\left\{\neg p: p \in \operatorname{enr}\left(\operatorname{ALT}\left([[T C L U R P]]_{D, w}\right)\right\}\right.$

Les conséquences de (18) sont que, pour chaque alternative, son enrichissement, qui asserte qu'aucun individu d'aucun sous-ensemble complémentaire ne vérifie la portée, est nié. Donc tous les individus de D vérifient la portée. Étant donné que D est un domaine qui traverse les différents mondes, on obtient une lecture assez improbable, selon laquelle les individus de différents mondes vérifient tous la phrase contenant le TCL-U. Par exemple Paul read any book signifierait que Paul a lu tous les livres possibles dans tous les mondes.

\footnotetext{
${ }^{8}$ Il semble que Chierchia cible des éléments qui ne sont pas en eux-mêmes des quantificateur universels, ce qui exclut des cas comme celui de tout. Cependant, il y a là une décision qui peut être discutée.
} 
Nous ferons trois objections à cette approche. D'abord, elle ne semble pas plus «justifiée » que des approches concurrentes, puisqu'il faut associer aux différents éléments les systèmes d'alternatives et d'enrichissements corrects. Il est donc difficile d'invoquer la généralité des phénomènes pragmatiques. En fait, pour que l'hypothèse découle de principes généraux, il faudrait montrer que ses aspects les plus spécifiques, notamment l'enrichissement des alternatives, existent pour d'autres phénomènes empiriques. Deuxièmement, nous soutenons que l'enrichissement des alternatives est contraire à l'esprit de la pragmatique Gricéenne, en dépit de ce que dit Chierchia. En effet, l'enrichissement ne fait sens que par rapport à une économie de la communication. C'est en comparant ce qui aurait pu être dit à ce qui a été effectivement dit qu'on peut reconstituer des stratégies de production et d'interprétation. Postuler un enrichissement pour les alternatives, qui, en tant qu'alternatives, n'ont pas été communiquées revient à tirer des conséquences inférentielles de ce qui n'a été ni dit ni implicité, ce qui nous paraît radicalement anti-Gricéen. Enfin, l'explication proposée par Chierchia pour justifier le caractère anormal des TCL-U sans subtrigging pose deux problèmes. L'un concerne le domaine $\mathrm{D}$. Rien n'empêche de choisir $\mathrm{D}$ pour lui donner une sorte de portée «métaphysique » qui devrait en théorie permettre l'emploi d'un TCL-U. Mais un exemple comme (19a) est très maladroit. De plus, Chierchia attribue au subtrigging la propriété de localiser les événements décrits, ce qui équivaut à une limitation de $\mathrm{D}$ qui l'empêcherait de couvrir plusieurs mondes. Cette solution, dont le principe (mais pas le détail) est dû à Dayal (1998), se heurte à des exemples où on localise spatio-temporellement la restriction sans constater d'amélioration notable (19b). Dayal $(1998,2005)$ avait d'ailleurs vu ce problème et restreint les constructions du type subtrigging, mais des exemples comme (19c), qui reprend (11c), montrent qu'il ne suffit pas d'avoir une construction avec deux prédications, càd une relative déterminative et le groupe verbal, ce qui facilite une interprétation conditionnelle.

(19) a. ??Hier, Dieu a créé n'importe quel monde

b. ??Tout billet du portefeuille est tombé

c. ??Tout étudiant qui était dans la pièce a entendu l'explosion

Il reste deux questions. D'une part, comment se fait-il que l'intuition d'élargissement soit souvent associée aux TCL ? Selon nous, il s'agit d'un effet pragmatique simple : puisqu'un TCL insiste sur l'équité, il met en valeur l'absence d'exceptions, qui violerait PAS-DEPERDANT. De l'absence d'exception on passe facilement à l'idée que le domaine est « élargi », c'est-à-dire qu'on inclut absolument tous les individus. D'autre part, pourquoi y a-t-il un lien entre polarité négative et TCL ? Il est tentant d'invoquer ici la scalarité, qui servirait de moteur à une quantification universelle, selon les principes décrits par Fauconnier (1977), Israël (2001). C'est notamment la base de l'analyse proposée par Horn (2000) pour any. Nous renvoyons à Larrivée (2007) et Muller (2007a) pour des études des relations entre scalarité et TCL en français. Nous adoptons la position de Muller (2007a) qui nuance fortement la supposée parenté entre scalarité et choix libre, en montrant qu'il s'agit bien souvent d'effets pragmatiques plus que d'une origine sémantique commune. Il est en revanche possible que l'équité, définie sur les individus qui satisfont des propriétés, soit un aspect particulier d'une propriété encore plus générale qui est la non-référence aux événements. Les contextes monotones-décroissants permettent de s'abstraire des événements précis. Par exemple, pour any, He didn't read any book ou Did he read any book? ne font pas référence à des événements de lecture ou de non-lecture. La possibilité de choix, ou l'interprétation contrefactuelle qui s'ajoute à l'interprétation épisodique dans certains cas, éloignent également la référence à des événements précis, soit parce que ceux-ci ne sont pas déterminés au moment de l'énonciation, soit parce qu'il existe des événements alternatifs eux-mêmes non 
déterminables. On voit donc qu'il est tentant de subsumer ces différents cas sous une notion qu'on pourrait décrire comme l'indépendance par rapport à un ensemble d'événements précis. C'est toutefois, pour le moment, un point qui doit être laissé à la spéculation.

\section{Les TCL : existentiels ou universels?}

Dans cette section nous aborderons deux points : premièrement, être un TCL n'implique pas être un indéfini existentiel, deuxièmement un TCL comme n'importe quel amène à s'interroger sur l'hypothèse du caractère fondamentalement existentiel des TCL qui ne sont pas des quantificateurs universels.

L'existence de tout montre qu'il n'est pas obligatoire d'être un indéfini existentiel pour être un TCL. Tout rencontre les problèmes typiques des TCL dans les phrases épisodiques et partage avec n'importe quel de nombreux environnements (Jayez et Tovena 2005), ce qui suggère a priori de le ranger dans les TCL. On pourrait objecter que, étant un quantificateur universel, il ne permet pas le choix. ${ }^{9}$ C'est bien ce qui se passe lorsqu'on a un domaine de restriction déterminé, mais cette impression disparaît lorsqu'on a un domaine variable. Ainsi (20a), qui fait référence à des voitures d'un ensemble déterminé, est anormal, alors que (20b), qui concerne un ensemble évolutif, est beaucoup plus naturel. Par rapport aux alternatives, on est avec (20b) dans une configuration qui respecte l'équité, puisque, dans les différentes évolutions futures du monde réel on peut imaginer des ensembles différents de voitures. Il n'y a donc pas lieu de compliquer l'hypothèse centrale.

(20) a. ??Contrôle toute voiture (du parking)

b. Contrôle toute voiture (qui se présentera)

On notera que, lorsqu'on a un TCL qui n'est un quantificateur universel, le choix d'un individu ou d'un ensemble n'est pas toujours déterminé indépendamment du contexte. Par exemple, si (21a) est utilisé dans le contexte d'un jeu télévisé où un candidat doit ouvrir une porte et révéler ce qui est caché derrière, on aura tendance à comprendre qu'il doit choisir une seule porte. Si en revanche on s'adresse à la police qui cherche un fuyard, on conçoit que la police puisse ouvrir toutes les portes. Seule la présence d'un pluriel impliquerait qu'on peut ouvrir n'importe quel sous-ensemble de portes, voire la totalité. En revanche, la latitude semble très limitée lorsque le TCL contient un : (21b) implique qu'une seule porte peut être ouverte.

(21) a. Vous avez le droit d'ouvrir n'importe quelle porte

b. Vous avez le droit d'ouvrir une porte quelconque / quelle qu'elle soit

Examinons à présent le cas de n'importe quel. Il semblerait qu'il ait la même distribution que la forme avec apposition, parallèle au supplementary any étudié par Horn (2000) Dayal (2004), le choix du singulier ou du pluriel étant fonction du contexte. Ainsi, (21a) serait paraphrasable par (22) . Lorsque n'importe quel $\mathrm{N}$ est en position objet, cette paraphrase s'étend aux impératifs et aux modaux de nécessité ou d'obligation (devoir). Elle paraît aussi s'étendre, mais moins facilement, aux phrases suscitant de préférence une interprétation épisodique.

(22) a. Vous avez le droit d'ouvrir une porte, n'importe laquelle

b. Vous avez le droit d'ouvrir des portes, n'importe lesquelles

\footnotetext{
${ }^{9}$ Cela n'implique pas que ce soit un quantificateur collectif. Au contraire, Kleiber \& Martin (1977) ont montré qu'il était distributif.
} 
En revanche, lorsque n'importe quel $\mathrm{N}$ est en position sujet, les données sont moins uniformes. Imaginons que le locuteur décrive la règle d'un jeu de cartes, et comparons différentes expressions (23).

(23) a. À ce stade, une carte, n'importe laquelle, peut être prise et placée dans l'autre paquet

b. À ce stade, une carte, n'importe laquelle, doit être prise et placée dans l'autre paquet

c. À ce stade, tu peux prendre n'importe quelle carte et la placer dans l'autre paquet

d. À ce stade, tu dois prendre n'importe quelle carte et la placer dans l'autre paquet

e. À ce stade, n'importe quelle carte peut être prise et placée dans l'autre paquet

f. ?? À ce stade, n'importe quelle carte doit être prise et placée dans l'autre paquet

Dans cette série, (23f) est de loin la phrase la plus problématique. On pourrait mettre cette impression sur le compte de la modalité. Il a été soutenu dans la littérature (voir par exemple Dayal 1998) que les TCL sont incompatibles avec les opérateurs modaux à force de quantification universelle ${ }^{10}$ (nécessité, obligation, croyance, probabilité, etc.). Cette généralisation est excessive. En fait, pour n'importe quel, des exemples comme (23d) ont deux interprétations. L'une fait jouer l'effet dépréciatif. L'interlocuteur est alors requis de prendre une carte sans effectuer aucun choix sérieux (au hasard). L'autre mobilise l'attitude du locuteur, qui signale qu'il ne pose aucun restriction sur le choix de la carte. Il est probable que la coexistence de ces deux interprétations complique le traitement, mais cela ne relève pas d'un conflit avec une préférence grammaticale.

Une explication possible du contraste illustré en (23) est que, en position sujet, n'importe quel $\mathrm{N}$ a une interprétation universelle, ce qui le rend analogue à tout et prédit une similitude de distribution dans cette position, qu'on observe effectivement dans les structures de type (23), voir (24a,b), et dans les interprétations génériques $(24 \mathrm{c}, \mathrm{d})$, où, par ailleurs, la forme avec apposition est un peu bizarre, voir (24).

(24) a. À ce stade, toute carte peut être prise et placée dans l'autre paquet

b. ??À ce stade, toute carte doit être prise et placée dans l'autre paquet

c. Tout flatteur vit aux dépens de celui qui l'écoute

d. N'importe quel flatteur vit au dépens de celui qui l'écoute

e. ?Un flatteur, n'importe lequel, vit au dépens de celui qui l'écoute

N'importe quel et tout sont également parallèles dans les comparatives, où la forme avec apposition n'est guère possible.

(25) a. Paul a mieux réussi que n'importe quel / tout autre candidat

b.??Paul a mieux réussi qu'un candidat, n'importe lequel

Nous concluons de ces remarques que n'importe quel peut être analysé comme existentiel ou universel en fonction de la structure dans laquelle il intervient, et que la discussion sur le caractère existentiel ou universel des TCL doit tenir compte de cette variabilité. Une étude plus approfondie devrait, si elle le peut, déterminer à quoi tient ce comportement protéiforme, où la fonction thématique pourrait jouer un rôle.

\section{Les comparatives}

\footnotetext{
${ }^{10}$ Ainsi nommés parce qu'ils impliquent que la proposition sur laquelle ils portent est vraie dans toutes les alternatives.
} 
Dans la littérature, lorsque l'on discute des TCL et des TPN, les comparatives restent un contexte problématique. Une première question est de savoir si on les traite comme une catégorie homogène, étant donné les différences de classification interne. Il y a en effet plusieurs types syntaxiques: les comparatives nominales (où le deuxième terme de la comparaison est un $\mathrm{SN}$ ) et les comparatives phrastiques (où le deuxième terme est une phrase). Le premier type est illustré en (26a) pour l'anglais. Le deuxième type est encore subdivisé en comparative phrastique entière et comparative phrastique réduite, illustrées respectivement en (26b) et (26c)

(26) a. Mary ran faster than any boy

b. Jim was less obnoxious than anyone at all before him had been

c. Mary ran faster than anybody expected

Selon Hoeksema (1983), aux différents types syntaxiques correspondent des propriétés sémantiques différentes qui se traduisent par une possibilité variable d'héberger des TCL et des TPN. Cet espace de variation est définissable en termes de monotonie. D'après Hoeksema, les comparatives nominales seraient un contexte qui légitime seulement des TCL, à preuve le fait que l'occurrence de any dans cet environnement n'a qu'une lecture universelle et peut donc être modifiée par almost, voir (27a). En revanche, les comparatives phrastiques seraient un environnement monotone décroissant qui légitime des TPN. Cette position est remise en cause par l'observation que le test de la modification par almost n'est pas décisif, car cet adverbe peut également modifier any dans des comparatives phrastiques, voir (27b). Toujours sous l'hypothèse que la monotonie est pertinente, la distinction adéquate serait celle entre les comparatives phrastiques entières et les nominales d'un côté, et les réduites de l'autre, selon Hendricks (1992), qui propose, pour sa part, un critère basé sur la nature de l'opérateur comparatif: more donnant une fonction décroissante, fewer une croissante ${ }^{11}$ et exactly une non monotone. Dans cette approche, la comparative de supériorité ou d'infériorité est analysée comme un tout à la façon d'un quantificateur généralisé.
a. Mary ran faster than almost any boy
b. This girl is smarter than almost any boy is

Les données du français peuvent-elles nous éclairer? Les exemples en (28) montrent que les comparatives nominales légitiment des TCL qui n'ont pas de sensibilité négative, par exemple n'importe quel et tout. Rappelons que, lorsque n'importe quel figure dans la portée immédiate de la négation, il ne se combine pas avec elle au sens où le ferait un existentiel comme un, qui a à peu près le comportement d'un quantificateur existentiel logique avec $\neg \exists=\forall \neg$. Pourtant la négation est un légitimeur de TPN par excellence et son effet sur un TPN est précisément ce type de combinaison. On n'a pas de différence notable avec les autres types de comparatives (phrastiques entières et réduites), voir (29).

(28) a. Cet accident a fait plus de morts que n'importe quel autre

b. Marie a mieux réussi que toute autre élève de sa classe

\footnotetext{
${ }^{11}$ Encore faudrait-il considérer la distinction entre lecture maximale et lecture minimale dans la comparative d'infériorité (distinction que Hendricks attribue à Rullman). Considérons (i) et ses deux paraphrases en français.

(i) Jim drove less fast than was permitted

Jim a conduit à une vitesse inférieure à la limite maximale permise

Jim a conduit à une vitesse inférieure à la limite minimale permise

La première paraphrase illustre la lecture maximale, ici le comparatif est monotone croissant. La deuxième illustre la lecture minimale, où le comparatif est monotone décroissant, lecture qui n'est disponible que dans certains cas.
} 
(29) a. Ce directeur a attaqué plus de membres du laboratoire que n'importe quel directeur avant lui ne l'a fait

b. Cette affaire a duré plus longtemps que n'importe quel politicien n'aurait imaginé

Ce que l'on peut dire à ce stade est que, si les comparatives sont associées à de la monotonie, il n'existe pas d'observation permettant de dire que la présence d'un TCL dans une comparative indique qu'un TCL a des propriétés de monotonie. De plus, l'hypothèse de départ apparaît fragile ; il ya des raisons de penser que les comparatives ne sont pas à analyser en termes de monotonie (voir Schwarzchild et Wilkinson 2002). D'une certaine manière, la discussion sur les propriétés de monotonie s'est poursuivie sans prendre vraiment en compte les conséquences du fait que les TCL sont appréciés indépendamment des items de polarité. Or, on constate que, d'une part, avec l'essor de l'étude des TCL à la fin des années 90, le découpage des données a parfois changé. De nos jours, il n'y a pas d'unanimité pour dire quelle partie de la distribution de any doit être attribuée à sa lecture CL et quelle autre à la polarité négative, indépendamment du fait que l'on postule deux any ou un seul. Par exemple, qu'est-ce qui prouve que l'occurrence de any en (26b) est un cas de polarité négative? Il n'y a aucun problème pour traduire la phrase en employant un TCL (Jean s'est révélé moins nuisible que n'importe qui sans exception l'avait été avant lui).

Une deuxième question ouverte porte sur la nature épisodique de ces phrases. Il semble que l'occurrence d'un TCL est d'autant plus naturelle que la phrase est interprétée comme exprimant une qualité qui explique la supériorité (ou l'infériorité) de l'individu dénoté par le groupe nominal sujet de la phrase. Ainsi l'exemple en (28b) ne fait pas directement référence à une série d'événements, par exemple les rencontres d'un tournoi. L'interprétation dominante est que la prestation globale de Marie était supérieure à celle de toutes ses collègues. Donc une lecture où on distribue le prédicat sur les éléments du deuxième terme et où on obtient une liste d'événements peut être dérivée mais ne peut pas être la seule possible.

Nous avons évoqué à la fin de la section 3 , à propos de la polarité négative, la possibilité de caractériser les TCL en termes de non-référence à des événements, plutôt qu'à des individus. Il n'est pas impossible que cette hypothèse se révèle pertinente dans ce cas. Une comparative décrit une relation entre deux mesures, qui s'abstrait des événements sur lesquels ces mesures se fondent. Si l'on raisonne en termes de degrés, (28b), par exemple assigne à Marie un degré supérieur au degré maximal de réussite de l'ensemble des autres filles. Bien que la façon dont ces degrés ont été déterminés relève d'événements particuliers, la comparaison des degrés, en elle-même, n'a rien d'un événement. On peut donc imaginer que c'est le lien intrinsèque entre comparatives et mesure qui sert de support à l'emploi des TCL, mais cette idée reste à mettre à l'épreuve de manière précise.

\section{Conclusions}

Dans cet article, nous avons abordé quatre questions importantes qui sont encore en discussion, malgré la littérature assez abondante consacrée aux déterminants et pronoms de choix libre. La première concerne les frontières mêmes de la notion de " choix libre ». Nous avons proposé de caractériser son noyau par une contrainte minimale d'équivalence dans une dimension, que nous avons détaillée dans une forme unifiée avant de montrer comment elle peut se diversifier selon les différents cas, par ajout de contraintes et ou par variation sur les dimensions possibles. La seconde question concerne la notion d'élargissement. D'après Kadmon et Landman (1993), any introduit une extension du domaine d'individus pertinents (widening). La discussion de cette proposition, qui semble intuitivement très satisfaisante 
pour les TCL, nous a permis de montrer que l'élargissement est un effet et pas un phénomène explicatif. La question suivante est celle de la valeur existentielle ou universelle des TCL. Nous avons argumenté en faveur d'une position nuancée. D'une part, même s'il y a une dominance typologique des TCL existentiels, il peut exister des TCL universels comme tout. D'autre part, il n'est pas évident que la distribution d'un TCL doive être uniformément existentielle ou universelle, un point que nous avons discuté à partir de l'exemple de n'importe quel. Enfin, nous avons examiné le cas des phrases comparatives qui constituent encore une question ouverte, car elles peuvent abriter des TCL même lorsqu'elles font référence à des événements particuliers, configuration qui a) rend généralement les mêmes TCL inappropriés ou b) leur fait adopter des valeurs restreintes (dépréciatives, par exemple) qu'on n'observe pas avec les comparatives.

\section{Références}

Béguelin Marie-José, 2002. Routines syntagmatiques et grammaticalisation : le cas des clauses en $n$ 'importe, in A. Leth Andersen et H. Nølke (eds), Macro-syntaxe et macrosémantique, Berne, Peter Lang, 43-69

Chierchia Gennaro, 2006. Broaden your views, Linguistic Inquiry 37, 535-590

Choi Jinyoung, 2007. Free choice and negative polarity: a compositional analysis of Korean polarity sensitive items, $\mathrm{PhD}$, University of Pennsylvania

Corblin Francis, 2004. Quelque, In Handbook of French semantics, ed. F. Corblin and H. de Swart. CSLI Publications, 99-107

Dayal, Veneeta,1998. Any as inherently modal. Linguistics and Philosophy 21, 353-422

Dayal, Veneeta, 2005. The universal force of free choice any, Linguistic Variation Yearbook $2004,5-40$

Falaus Anamaria, 2009, Items de polarité et indéfinis dépendants en roumain, Thèse de Doctorat, Université de Nantes

Fauconnier Gilles, 1977. Polarité syntaxique et sémantique, Linguisticae Investigationes 1, 138

Giannakidou Anastasia, 1997. The landscape of polarity items, $\mathrm{PhD}$, University of Groningen, The Netherlands.

Giannakidou Anastasia, 2001. The meaning of free choice. Linguistics and Philosophy 24, 659-735

Haspelmath Martin, 1997. Indefinite pronouns. Oxford: Oxford University Press.

Hendriks Petra, 1995. Comparatives and Categorial Grammar, PhD, University of Groningen

Hoeksema Jacob, 1983. Negative polarity and the comparative, Natural language and linguistic theory : 1, 403-434

Horn Laurence, 2000. Any and (-)ever: Free choice and free relatives. In Proceedings of the 15th Annual Conference of the Israeli Association for Theoretical Linguistics, 71-111

Israël Michael, 2001. Minimizers, Maximizers and the Rhetoric of Scalar Reasoning, Journal of Semantics 18, 297-331

Jayez Jacques et Lucia M. Tovena, 2002. Determiners and uncertainty. in Proceedings of SALT 12, ed. B. Jackson, 71-111. Ithaca, NY, CLC Publications

Jayez Jacques et Lucia M. Tovena, 2003. Any and eventualities, in Proceedings of the Fourteenth Amsterdam Colloquium, 151-156

Jayez Jacques et Lucia M. Tovena, 2005a. Free choiceness and non-individuation. Linguistics and Philosophy 28, 1-71

Jayez Jacques et Lucia M. Tovena, 2005b. When 'widening' is too narrow, in Proceedings of the Fifteenth Amsterdam Colloquium, 131-136

Jayez Jacques et Lucia M. Tovena, 2006. Epistemic determiners, Journal of Semantics 23, 
217-250

Jayez Jacques et Lucia M. Tovena, 2007. Subtrigging as alternatives through regularities, in Proceedings of the Sixteenth Amsterdam Colloquium, 127-132

Jayez Jacques et Lucia M. Tovena, 2008. Evidentiality and Determination, in Proceedings of Sinn und Bedeutung 12, 271-286

Kadmon Nirit, et Fred Landman, 1993. Any. Linguistics and Philosophy 4, 353-422

Kleiber Georges et Robert Martin, 1977. La quantification universelle en Français, Semantikos 2, 19-36

Larrivée Pierre, 2007. La scalarité d'indéfinis à sélection arbitraire. Travaux de Linguistique $54,97-107$

LeGrand, Jean E., 1975. Or and any: The semantics and syntax of two logical operators. $\mathrm{PhD}$, University of Chicago

Muller Claude, 2006. Polarité négative et free choice dans les indéfinis de type que ce soit et n'importe, Langages 162, 7-31

Muller Claude, 2007a. Les indéfinis free choice confrontés aux explications scalaires, Travaux de linguistique 54, 83-96

Muller Claude, 2007b. Quelque, déterminant singulier, Cahiers de lexicologie 90-1, 135-149

Paillard Denis, 1997. N'importe qui, n'importe quoi, n'importe quel N. Langue française 116, $100-114$

Pescarini Sandrine, 2009. Analyse synchronique et diachronique de l'item à choix libre n'importe quel : comparaison avec tout, Thèse de Doctorat, Université Nancy 2

Sauerland Uli, 2005. The epistemic step, talk delivered at "Experimental pragmatics », Cambridge.

Schwarzschild Roger et Karina Wilkinson. 2002. Quantifiers in comparatives, Natural Language Semantics 10, 1-41

Tovena Lucia M., 2000, Neg-raising : negation as failure?, in Perspectives on Negation and polarity items, Hoeksema J. et al ; (eds.) John Benjamins, 331-356

Tovena Lucia M., Déprez Viviane, and Jayez Jacques. 2005. Polarity sensitive items, In Handbook of French semantics, ed. F. Corblin and H. de Swart. CSLI Publications

Tovena, Lucia M. et Jacques Jayez, 1997. Any as a Finian quantifier, in Proceedings of the Eleventh Amsterdam Colloquium, 295-300

Vlachou Evangelia, 2007. Free choice in and out of context: semantics and distribution of French, Greek and English Free Choice Items. PhD, Université Paris 4 et Université d'Utrecht 